PROCEEDINGS OF THE

AMERICAN MATHEMATICAL SOCIETY

Volume 133, Number 10, Pages 2949-2955

S 0002-9939(05)08153-0

Article electronically published on May 9, 2005

\title{
GENERALIZED SUBDIFFERENTIAL OF THE DISTANCE FUNCTION
}

\author{
S. DUTTA
}

(Communicated by Jonathan M. Borwein)

\begin{abstract}
We derive the proximal normal formula for almost proximinal sets in a smooth and locally uniformly convex Banach space. Our technique leads us to show the generic Fréchet smoothness of the distance function in the case the norm is Fréchet smooth, and we derive a necessary and sufficient condition for the convexity of a Chebyshev set in a Banach space $X$ with norms on $X$ and $X^{*}$ locally uniformly convex.
\end{abstract}

\section{INTRODUCTION}

Let $X$ be a real Banach space. The closed unit ball of $X$ will be denoted by $X_{1}$. For a closed set $K$ in $X$ and $x \in X$, we denote the distance function of $K$ at $x$ by $d_{K}(x)=\inf \{\|x-k\|: k \in K\} . d_{K}$ is a 1-Lipschitz function on $X$. The metric projection of $x$ onto $K$ is $P_{K}(x)=\left\{k \in K:\|x-k\|=d_{K}(x)\right\}$. The set $K$ is called proximinal (Chebyshev) if for every $x \in X \backslash K, P_{K}(x)$ is nonempty (singleton). $K$ will be called almost proximinal if $P_{K}(x)$ is nonempty for a dense set of $x \in X \backslash K$.

Let $h: X \rightarrow \mathbb{R}$ be a Lipschitz function. For $x, y \in X$ we define

$$
h^{0}(x, y)=\limsup _{z \rightarrow x, t \rightarrow 0^{+}} \frac{h(z+t y)-h(z)}{t}
$$

and the generalized subdifferential of $h$ at $x \in X$ is defined as

$$
\partial h(x)=\left\{f \in X^{*}: f(y) \leq h^{0}(x, y) \forall y \in X\right\} .
$$

Given a nonempty closed set $K$ in $X$ and the distance function $d_{K}$ at $x$ there is a geometrical object called the normal cone at $x$ which is defined as

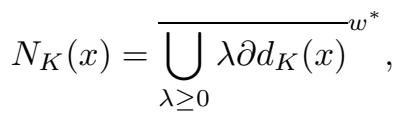

the $w^{*}$-closed convex cone generated by $\partial d_{K}(x)$. In $\mathbb{R}^{n}$, the generalized subdifferential of $d_{K}$ at $x \in b d y K$ has a geometrical formulation as the convex hull of the origin and the cluster points of $\frac{v_{n}}{\left\|v_{n}\right\|}$ where $v_{n} \perp K$ at points $x_{n} \in b d y K$ as $x_{n} \rightarrow x$ and $\left\|v_{n}\right\| \rightarrow 0$. The corresponding expression for the normal cone $N_{K}(x)$ in terms of approximating normals to $K$ in $\mathbb{R}^{n}$ is called the proximal normal formula.

Received by the editors April 10, 2003.

2000 Mathematics Subject Classification. Primary 41A65, 41A52; Secondary 46B20.

Key words and phrases. Nearest points, proximinal sets, almost proximinal sets, Chebyshev sets, generalized subdifferential. 
The best known infinite-dimensional formulation of the proximal normal formula in Banach space was given by Borwein and Giles in [1]. In particular they derived the formula for two different cases: For an almost proximinal set using uniform Gâteux smoothness of norm on $X$ and for nonempty closed sets in a smooth reflexive Banach space with Kadec norm.

In the present note, we show that one can obtain the proximal normal formula for an almost proximinal set if the norm on $X$ is smooth and locally uniformly convex. Our technique is based on observing density of the set $E_{1}(K)$, where $E_{1}(K)$ denotes the set of points in $X \backslash K$ for which every minimizing sequence in $K$ converges to a unique nearest point. In the first section, we show that in a smooth Banach space if $E_{1}(K)$ is dense, then we can give an explicit description of the generalized subdifferential of the distance function and thereby obtain the proximal normal formula. In the second section, we show that for any almost proximinal set $K$ in $X$, a sufficient condition for $E_{1}(K)$ to be dense is the local uniform convexity (LUR) of the norm on $X$.

Differentiability properties of the distance function had been of great interest in studies in optimization theory as it relates to the famous problem of convexity of Chebyshev sets. The landmark theorem in this direction is that of Vlasov [5]: If $X^{*}$ is strictly convex, then every Chebyshev set in $X$ with continuous metric projection is convex. Fitzpatrick [4] observed that in a Banach space with both the norms on $X$ and $X^{*}$ Fréchet smooth, a differentiability condition on the distance function implies the convexity of Chebyshev sets. Note that Fréchet smoothness of the norm on $X^{*}$ already implies reflexivity of $X$. Here we show that our technique leads to generic (Fréchet) smoothness of the distance function in the case the norm is (Fréchet) smooth. This improves a lot on the result of [1] where the authors derived it for Hilbert spaces, and this helps us to give a necessary and sufficient condition for the convexity of a Chebyshev set in Banach spaces where the norms on $X$ and $X^{*}$ are LUR.

\section{The PROXIMAL NORMAL FORMULA}

We take $E(K)$ to be the set of points in $X \backslash K$ which has nearest points in $K$ and $E_{1}(K)$ to be the set $x \in E(K)$ such that every minimizing sequence for $x$ in $K$ converges to a unique nearest point of $x$. By $\mathcal{D}$ we will mean the duality map on $X$, that is, $\mathcal{D}(x)=\left\{f \in X_{1}^{*}: f(x)=\|x\|\right\}$. For explanations of the properties of the generalized subdifferential we use in this note, see [2].

Lemma 1. Let $x \in E_{1}(K)$. Then

$$
\partial d_{K}(x) \subseteq \mathcal{D}\left(x-P_{K}(x)\right) .
$$

Equality holds if the norm on $X$ is smooth at $x-P_{K}(x)$. Moreover, if the norm on $X$ is Fréchet smooth at $x-P_{K}(x), d_{K}$ is Fréchet smooth at $x$.

Proof. Let $f \in \partial d_{K}(x)$. Since $\mathcal{D}\left(x-P_{K}(x)\right)$ is a $w^{*}$-compact convex set, it is enough to show that given any $y \in X,\|y\|=1$, there is a $g \in \mathcal{D}\left(x-P_{K}(x)\right)$ such that $f(y) \leq g(y)$.

By definition of $\partial d_{K}(x)$, given any $\varepsilon>0$ there exist $z_{n} \rightarrow x$ and $t_{n} \rightarrow 0^{+}$such that

$$
f(y)-\varepsilon \leq \frac{d_{K}\left(z_{n}+t_{n} y\right)-d_{K}\left(z_{n}\right)}{t_{n}} .
$$


Get $k_{n} \in K$ such that $\left\|z_{n}-k_{n}\right\|<d_{K}\left(z_{n}\right)+t_{n}^{2}$. Then $\left\{k_{n}\right\}$ is minimizing for $x$ as well and hence $k_{n} \rightarrow P_{K}(x)$. Now

$$
f(y)-\varepsilon<\frac{\left\|z_{n}+t_{n} y-k_{n}\right\|-\left\|z_{n}-k_{n}\right\|+t_{n}^{2}}{t_{n}} .
$$

By the mean value property of subdifferentials (see [2, Theorem 2.3.7]), there exist $g_{n} \in \mathcal{D}\left(w_{n}\right)$ such that $g_{n}\left(t_{n} y\right)=\left\|z_{n}+t_{n} y-k_{n}\right\|-\left\|z_{n}-k_{n}\right\|$, where $w_{n}$ lies on the line $\left[z_{n}-k_{n}, z_{n}+t_{n} y-k_{n}\right]$. Thus $f(y)-\varepsilon<g_{n}(y)+t_{n}$. Let $g$ be a $w^{*}$-cluster point of $\left\{g_{n}\right\}$. Since $w_{n} \rightarrow x-P_{K}(x)$ in norm, by the upper semicontinuity of $\mathcal{D}$, we have $g \in \mathcal{D}\left(x-P_{K}(x)\right)$ and $f(y)-\varepsilon \leq g(y)$. Thus we have the desired result.

If the norm on $X$ is smooth at $x-P_{K}(x), \mathcal{D}\left(x-P_{K}(x)\right)$ is a singleton and so is $\partial d_{K}(x)$. Note that this implies $d_{K}$ is smooth at $x$.

If the norm is Fréchet smooth at $x-P_{K}(x)$, note that for $f \in \partial d_{K}(x)$ and any $z \in X,\|z\|=1$, we have

$$
f(z)=\lim _{n \rightarrow \infty} \frac{d_{K}\left(x+\frac{1}{n} z\right)-d_{K}(x)}{1 / n} \leq \lim _{n \rightarrow \infty} \frac{\left\|x-P_{K}(x)+\frac{1}{n} z\right\|-\left\|x-P_{K}(x)\right\|}{1 / n}
$$

where the right-hand side converges to $f(z)$ uniformly over all $\|z\|=1$. Thus $d_{K}$ is Fréchet smooth at $x$.

Let $E_{1}(K)$ be dense in $X \backslash K$. For $x \in X \backslash K$, denote by $D_{x}(K)$ the $w^{*}$-cluster points of $\mathcal{D}\left(y_{n}-P_{K}\left(y_{n}\right)\right)$, where $y_{n} \in E_{1}(K)$ and $y_{n} \rightarrow x$. Our main result in this section is the following:

Theorem 2. Let $X$ be smooth. Let $K$ be a closed set in $X$ such that $E_{1}(K)$ is dense in $X \backslash K$. Then for any $x \in X \backslash K$ we have

$$
\partial d_{K}(x)=\overline{c o} w^{*}\left\{D_{x}(K)\right\} .
$$

Thus we have a proximal normal formula in $X$ for $X$ smooth. This reads as follows:

Suppose $K$ is a closed set in $X$ such that $E_{1}(K)$ is dense in $X \backslash K$. Then for $x \in b d y K, N_{K}(x)$ is the $w^{*}$-closed convex cone generated by the origin and $D_{x}(K)$.

Proof. Let $f \in D_{x}(K)$. Then there is a sequence $\left\{y_{n}\right\} \in E_{1}(K), y_{n} \rightarrow x, f_{n} \in$ $\mathcal{D}\left(y_{n}-P_{K}\left(y_{n}\right)\right)$ such that $f_{n} \rightarrow f$ in the $w^{*}$-topology. By Lemma $1 f_{n} \in \partial d_{K}\left(y_{n}\right)$. By upper semicontinuity of $\partial d_{K}$, we have $f \in \partial d_{K}(x)$. Since $\partial d_{K}(x)$ is a $w^{*}$-closed convex set, $\overline{c o}^{w^{*}}\left\{D_{x}(K)\right\} \subseteq \partial d_{K}(x)$.

Conversely, let $f \in \partial d_{K}(x)$. As before, it is enough to show that for any $y \in X$, $\|y\|=1$, there exists $g \in \overline{c o}^{w^{*}}\left\{D_{x}(K)\right\}$ such that $f(y) \leq g(y)$.

Given $\varepsilon>0$ there are $z_{n} \in X \backslash K, z_{n} \rightarrow x$ and $t_{n} \rightarrow 0^{+}$such that for each $n$,

$$
f(y)-\varepsilon / 2 \leq \frac{d_{K}\left(z_{n}+t_{n} y\right)-d_{K}\left(z_{n}\right)}{t_{n}} .
$$

Choose $y_{n} \in E_{1}(K)$ such that $\left\|z_{n}+t_{n} y-y_{n}\right\|<t_{n}^{2}$. Then $d_{K}\left(z_{n}+t_{n} y\right) \leq$ $d_{K}\left(y_{n}\right)+t_{n}^{2}$ and $d_{K}\left(z_{n}\right)>d_{K}\left(y_{n}-t_{n} y\right)-t_{n}^{2}$. Thus for all sufficiently large $n$,

$$
f(y)-\varepsilon / 2 \leq \frac{d_{K}\left(y_{n}\right)-d_{K}\left(y_{n}-t_{n} y\right)}{t_{n}}+2 t_{n} \leq d_{K}^{0}\left(y_{n}, y\right)+\varepsilon / 2+2 t_{n} .
$$

Since by Lemma 1, $\partial d_{K}\left(y_{n}\right)=\mathcal{D}\left(y_{n}-P_{K}\left(y_{n}\right)\right)$ is a singleton we have $d_{K}^{0}\left(y_{n}, y\right)=$ $g_{n}(y)$ where $g_{n}\left(y_{n}-P_{K}\left(y_{n}\right)\right)=\left\|y_{n}-P_{K}\left(y_{n}\right)\right\|$. Let $g$ be a $w^{*}$-cluster point of $g_{n}$. We then have $f(y)-\varepsilon \leq g(y)$ and the result follows. 


\section{Density of $E_{1}(K)$}

In this section we investigate sufficient conditions on $X$ such that for every almost proximinal set $K, E_{1}(K)$ is dense in $X \backslash K$. As mentioned in the introduction, this sufficient condition turns out to be local uniform convexity (LUR) of the norm.

In the following proposition we collect some properties of the set $E_{1}(K)$. Given $x \in X \backslash K$ and $\delta>0$ we define $P_{K}(x, \delta)=\left\{k \in K:\|x-k\|<d_{K}(x)+\delta\right\}$.

Proposition 3. Suppose $K$ is a closed set in $X$.

(a) $x \in E_{1}(K)$ if and only if given any $\varepsilon>0$ there is $a \delta>0$ such that diameter $P_{K}(x, \delta)<\varepsilon$.

(b) If $x \in E_{1}(K)$, then the metric projection $P_{K}$ is single-valued and continuous at $x$.

(c) $E_{1}(K)$ is a $G_{\delta}$ in $X$.

Proof. (a). This follows from the definition of $E_{1}(K)$.

(b). Again, $P_{K}$ is a singleton follows from the definition. To show continuity, let $x_{n} \rightarrow x$. Then any $k_{n} \in P_{K}\left(x_{n}\right)$ is a minimizing sequence for $x$ as well and hence converges to $P_{K}(x)$.

(c). For each $n \geq 1$, consider the set $G_{n}=\{x \in X \backslash K$ : there exists $\delta>0$, such that diameter $\left.P_{K}(x, \delta)<1 / n\right\}$. From $(a)$ it follows that $E_{1}(K)=\bigcap_{n \geq 1} G_{n}$. We need to show each $G_{n}$ is open.

Let $x \in G_{n}$. Thus there is a $\delta>0$ such that $\operatorname{dia} P_{K}(x, \delta)<1 / n$. Choose $0<\alpha<\delta / 2$ and $\beta=\delta-2 \alpha$. Then for $y \in X \backslash K,\|y-x\|<\alpha$ and $k \in P_{K}(y, \beta)$ we have $\|x-k\|<\|y-k\|+\alpha<d_{K}(y)+\beta+\alpha<d_{K}(x)+\beta+2 \alpha=d_{K}(x)+\delta$. Thus $P_{K}(y, \beta) \subseteq P_{K}(x, \delta)$ and thus $y \in G_{n}$ as well. This shows $G_{n}$ is open.

We now present our main theorem of this section:

Theorem 4. Let $X$ be a Banach space with LUR norm and $K$ be a closed almost proximinal set in $X$. Then $E_{1}(K)$ is a dense $G_{\delta}$ in $X \backslash K$.

Remark 5. In conjunction with Theorem 2 we observe that if the norm on $X$ is both LUR and smooth, for every almost proximinal set $K$ we have the proximal normal formula.

The proof of the following corollary follows from Lemma 1 and Proposition 3 In Theorem 10 of [1] the authors showed this for Hilbert spaces.

Corollary 6. Let the norm on $X$ be LUR and (Fréchet) smooth. Then the distance function generated by an almost proximinal set $K$ is generically (Fréchet) smooth on $X \backslash K$.

The proof of Theorem 4 is based on the observation that if we consider our set $K$ to be $X$ minus the open unit ball, then any point in the open unit ball has a nearest point on the unit sphere and if the space is LUR, for any such point except the origin, every minimizing sequence converges.

Proof of Theorem [4. By Proposition 3 it suffices to show that $E_{1}(K)$ is dense in $E(K)$. So, let $x \in E(K)$ and $k_{0} \in P_{K}(x)$. We show that given any $0<\varepsilon<1 / 3$, the point $x_{0}=x-\varepsilon\left(x-k_{0}\right) \in E_{1}(K)$.

We note that $d_{K}\left(x_{0}\right)=(1-\varepsilon) d_{K}(x)$. Let $\left\{k_{n}\right\}$ be a minimizing sequence for $x_{0}$. It is easy to observe that $\left\|x-k_{n}\right\| \rightarrow d_{K}(x)$ as well. Now, $\left\|x_{0}-k_{n}\right\| \rightarrow d_{K}\left(x_{0}\right)$, 
that is,

$$
\left\|k_{n}-x+\varepsilon\left(x-k_{0}\right)\right\| \rightarrow(1-\varepsilon) d_{K}(x) .
$$

Since

$$
\begin{aligned}
1-\varepsilon & \leq\left\|\frac{k_{n}-x}{\left\|x-k_{n}\right\|}-\varepsilon \frac{k_{0}-x}{d_{K}(x)}\right\| \\
& \leq \frac{1}{d_{K}(x)}\left\|k_{n}-x-\varepsilon\left(k_{0}-x\right)\right\|+\left\|k_{n}-x\right\|\left[\frac{1}{d_{K}(x)}-\frac{1}{\left\|x-k_{n}\right\|}\right],
\end{aligned}
$$

we have

$$
\left\|\frac{k_{n}-x}{\left\|x-k_{n}\right\|}-\varepsilon \frac{k_{0}-x}{d_{K}(x)}\right\| \rightarrow 1-\varepsilon .
$$

Let $u_{n}=\left(k_{n}-x\right) /\left\|x-k_{n}\right\|, u_{0}=\left(k_{0}-x\right) / d_{K}(x)$ and $\lambda=(1-2 \varepsilon) /(1-\varepsilon)$. Note that, since $e<1 / 3,1 / 2<\lambda<1$. Then $\left\|u_{n}\right\|=\left\|u_{0}\right\|=1$ and

$$
\left\|2 u_{n}-\left[\lambda u_{n}+(1-\lambda) u_{0}\right]\right\| \rightarrow 1 \text {. }
$$

Since

$$
\left\|2 u_{n}-\left(\lambda u_{n}+(1-\lambda) u_{0}\right)\right\| \geq 2-\left\|\lambda u_{n}+(1-\lambda) u_{0}\right\| \geq 1
$$

we have $\left.\| \lambda u_{n}+(1-\lambda) u_{0}\right) \| \rightarrow 1$ as well. Let

$$
f_{n}(\lambda)=1-\left\|\lambda u_{n}+(1-\lambda) u_{0}\right\| .
$$

Using convexity of the norm, we get that

$$
\left\|\lambda u_{n}+(1-\lambda) u_{0}\right\| \leq(2-2 \lambda)\left\|\frac{u_{n}+u_{0}}{2}\right\|+(2 \lambda-1) .
$$

It follows that

$$
f_{n}(\lambda) \geq(2-2 \lambda) f_{n}(1 / 2) \geq 0 .
$$

Since $f_{n}(\lambda) \rightarrow 0$, we have that $f_{n}(1 / 2) \rightarrow 0$, that is, $\left\|u_{n}+u_{0}\right\| \rightarrow 2$. Since $X$ is LUR, $u_{n} \rightarrow u_{0}$ and hence, $k_{n} \rightarrow k$.

We conclude this note with a result on the continuity of metric projection on Chebyshev sets. Our result, in conjunction with the result of Vlasov quoted in the introduction, gives a necessary and sufficient condition for convexity of Chebyshev sets in a Banach space $X$ such that both $X$ and $X^{*}$ are LUR. We believe this improves upon the known results in this direction.

Proposition 7. Suppose the norm on $X$ is both LUR and Fréchet smooth. Then for a Chebyshev set $K \subseteq X$ and $x \in X \backslash K$, the metric projection $P_{K}$ is continuous at $x$ if and only if $\partial d_{K}(x)$ is a singleton.

Proof. Let $K \subseteq X$ be a Chebyshev set and $x \in X \backslash K$ be such that $P_{K}$ is continuous at $x$. From Theorem 2 and Theorem 4 we have $\partial d_{K}(x)=\overline{c o}^{w^{*}}\left\{D_{x}(K)\right\}$.

Now, let $f \in D_{x}(K)$. By definition of $D_{x}(K)$, there exists $\left\{x_{n}\right\} \subseteq E_{1}(K)$, $x_{n} \rightarrow x$ and $f_{n} \in \mathcal{D}\left(x_{n}-P_{K}\left(x_{n}\right)\right)$ such that $f_{n} \rightarrow f$ in the $w^{*}$-topology. But by continuity of $P_{K}, x_{n}-P_{K}\left(x_{n}\right) \rightarrow x-P_{K}(x)$. Therefore, $f \in \mathcal{D}\left(x-P_{K}(x)\right)$. That is, $D_{x}(K) \subseteq \mathcal{D}\left(x-P_{K}(x)\right)$. Hence, $\partial d_{K}(x)=\overline{c o}^{w^{*}}\left\{D_{x}(K)\right\} \subseteq \mathcal{D}\left(x-P_{K}(x)\right)$ as well. Now, since $X$ is smooth, $\partial d_{K}(x)$ must be a singleton.

Conversely, let $K \subseteq X$ be a Chebyshev set and $x \in X \backslash K$ be such that $\partial d_{K}(x)$ is a singleton. By [1, Lemma 1], this implies $\partial d_{K}(x) \subseteq \mathcal{D}\left(x-P_{K}(x)\right)$. Since $X$ is smooth, we actually have $\partial d_{K}(x)=\mathcal{D}\left(x-P_{K}(x)\right)$.

Now, let $y_{n} \in X \backslash K, y_{n} \rightarrow x$. We want to show $P_{K}\left(y_{n}\right)$ converges to $P_{K}(x)$. Define $x_{n}=y_{n}-\frac{1}{n}\left(y_{n}-P_{K}\left(y_{n}\right)\right)$. By the proof of Theorem $4, x_{n} \in E_{1}(K)$ and 
$P_{K}\left(x_{n}\right)=P_{K}\left(y_{n}\right)$ for all $n>3$. Note that $x_{n} \rightarrow x$ as well and by Theorem [1] $\partial d_{K}\left(x_{n}\right) \subseteq \mathcal{D}\left(x_{n}-P_{K}\left(x_{n}\right)\right)$. Let $f_{n} \in \partial d_{K}\left(x_{n}\right)$ and let $f$ be a $w^{*}$-cluster point of $f_{n}$. Then by upper semicontinuity of $\partial d_{K}, f \in \partial d_{K}(x)=\mathcal{D}\left(x-P_{K}(x)\right)$. Since $X$ is Fréchet smooth, this would imply that $f_{n} \rightarrow f$ in norm as well. Hence,

$$
f\left(\frac{x_{n}-P_{K}\left(x_{n}\right)}{\left\|x_{n}-P_{K}\left(x_{n}\right)\right\|}\right) \rightarrow 1 .
$$

Now since the norm on $X$ is LUR, $f$ strongly exposes $\frac{x-P_{K}(x)}{\left\|x-P_{K}(x)\right\|}$. Thus

$$
\frac{x_{n}-P_{K}\left(x_{n}\right)}{\left\|x_{n}-P_{K}\left(x_{n}\right)\right\|} \rightarrow \frac{x-P_{K}(x)}{\left\|x-P_{K}(x)\right\|} .
$$

Since $\left\|x_{n}-P_{K}\left(x_{n}\right)\right\|=d_{K}\left(x_{n}\right) \rightarrow d_{K}(x)=\left\|x-P_{K}(x)\right\|$, we have $P_{K}\left(x_{n}\right)=$ $P_{K}\left(y_{n}\right) \rightarrow P_{K}(x)$ as desired.

Theorem 8. Suppose the norms on $X$ and $X^{*}$ are LUR. Then a Chebyshev set $K$ is convex in $X$ if and only if $\partial d_{K}(x)$ is a singleton for all $x \in X \backslash K$.

Proof. If $K$ is convex, then $\partial d_{K}$ coincides with the usual subdifferential of $d_{K}$, and if the norm on $X^{*}$ is LUR, then $d_{K}$ is Fréchet smooth at each $x \in X \backslash K$ (see [3] page 365]). Thus $\partial d_{K}(x)$ is a singleton for each such $x$.

Conversely, let $\partial d_{K}(x)$ be a singleton for each $x \in X \backslash K$. By Proposition [7, we have that the metric projection on $K$ is continuous. Thus by Vlasov's Theorem, $K$ is convex.

Remark 9. (a) $d_{K}$ being a Lipschitz function, the condition $\partial d_{K}(x)$ is a singleton for all $x \in X \backslash K$ reduces to strict differentiability of $d_{K}$ (see [2, page 30 for definition and Proposition 2.2.4 for the equivalence of these two). In particular, this is satisfied when $d_{K}$ is continuously differentiable on $X \backslash K$.

(b) In [4, Theorem 3.6], the author showed that for a closed set $K$ in a Banach space $X$ with the norms of $X$ and $X^{*}$ Fréchet differentiable, if for each $x \in X \backslash K$ there exists a unit vector $u \in X$ such that the directional derivative $D_{u} d_{K}(x)=1$, then $K$ is convex. A close look at the proof given in that paper actually shows that this condition implies $E_{1}(K)=X \backslash K$ and thus the set is Chebyshev and by Lemma 1 we also have that $\partial d_{K}(x)$ is a singleton for each $x \in X \backslash K$. Thus the result follows as a simple corollary of Theorem 8 .

\section{ACKNOWLEDGEMENT}

The results presented in this paper have been included in the author's Ph.D. thesis. The author would like to thank his thesis advisor, Dr. Pradipta Bandyopadhyay of Indian Statistical Institute, Kolkata. We also thank the referee for his careful examination and comments which improved the materials presented in this paper.

\section{REFERENCES}

[1] J. M. Borwein and J. R. Giles, The proximal normal formula in Banach space, Trans. Amer. Math. Soc. 302, No. 1, 1987, 371-381. MR0887515 (88m:49013)

[2] F. H. Clarke, Optimization and nonsmooth analysis, Canadian Math. Soc. series and monographs and advanced texts, Wiley, New York, 1983. MR0709590 (85m:49002)

[3] R. Deville, G. Godefroy, and V. Zizler, Smoothness and renormings in Banach spaces, Pitman Monographs and Surveys in Pure and Applied Mathematics, 64. Longman Scientific \& Technical, Harlow; 1993. MR,1211634 (94d:46012) 
[4] S. Fitzpatrick, Differentiation of real-valued functions and continuity of metric projections, Proc. Amer. Math. Soc. 91, No. 4, 1984, 544-548. MR.0746087 (85m:46039)

[5] L. P. Vlasov, Almost convexity and Chebyshev sets, Math. Notes. Acad. Sci. USSR 8, 1970, 776-779. MR0276736 (43:2476)

Stat-Math Division, Indian Statistical Institute, 203, B. T. Road, Kolkata 700108 , INDIA

E-mail address: sudipta_r@isical.ac.in

Current address: Department of Mathematics, Ben-Gurion University of the Negev, P.O.B. 653, Beer-Sheva 84105, Israel

E-mail address: sudipta@math.bgu.ac.il 